\title{
On rational approximations of the
}

\section{exponential function at rational points}

\section{Kurt Mahler}

Let $p, q, u$, and $v$ be any four positive integers, and let further $\delta$ be a number in the interval $0<\delta \leq 2$. In this note an effective lower bound for $q$ will be obtained which insures that

$$
\left|e^{u / v}-\frac{p}{q}\right|>q^{-(2+\delta)}
$$

In the special case when $u=v=1$, it was shown by $J$. Popken, Math. Z. 29 (1929), 525-541, that

$$
\left|e-\frac{p}{q}\right|>q^{-\{2+(c / \log \log q)\}} \text { for } q \geq C \text {. }
$$

Here $c$ and $C$ are two positive absolute constants which, however, were not determined explicity. A similarly noneffective result was given in my paper, $J$. reine angew. Math. 166 (1932), 118-150.

The method of this note depends again on the classical formulae by Hermite which I applied also op. cit.

1.

Denote by $m$ and $n$ two non-negative integers and put

$$
F(w)=\frac{w^{m}(w-1)^{n}}{m ! n !} \text { and } F(z ; w)=\sum_{k=0}^{\infty} z^{-k-1}\left(\frac{d}{d w}\right)^{k} F(w) \text {. }
$$

A simple calculation shows that

Received 19 December 1973. 


$$
F(z ; 0)=\sum_{k=m}^{m+n} z^{-k-1}\left(\begin{array}{l}
k \\
m
\end{array}\right) \frac{(-1)^{m+n-k}}{(m+n-k) !} \text { and } F(z ; 1)=\sum_{k=n}^{m+n} z^{-k-1}\left(\begin{array}{l}
k \\
n
\end{array}\right) \frac{1}{(m+n-k) !}
$$

Put therefore

$$
P(z)=(m+n) ! z^{m+n+1} E(z ; 0) \text { and } Q(z)=(m+n) ! z^{m+n+1} F(z ; 1) .
$$

Then

$$
P(z)=\sum_{k=m}^{m+n} k !(-1)^{m+n-k}\left(\begin{array}{l}
k \\
m
\end{array}\right)\left(\begin{array}{c}
m+n \\
k
\end{array}\right) z^{m+n-k}, Q(z)=\sum_{k=n}^{m+n} k !\left(\begin{array}{l}
k \\
n
\end{array}\right)\left(\begin{array}{c}
m+n \\
k
\end{array}\right) z^{m+n-k} .
$$

By these formulae, $P(z)^{\circ}$ and $Q(z)$ are polynomials in $z$ of the degrees $n$ and $m$, and with integral coefficients divisible by $m$ ! and $n !$, respectively.

It also follows from the definitions of $F(w)$ and $F(z ; w)$ that

$$
\int_{0}^{1} F(w) e^{-z w} d w=F(z ; 0)-F(z ; 1) e^{-z}
$$

Hence, on putting

$$
R(z)=(m+n) ! z^{m+n+1} e^{z} \int_{0}^{1} F(w) e^{-z w} d w,
$$

we obtain Hermite's identity

$$
P(z) e^{z}-Q(z)=R(z)
$$

2 .

From now on denote by $r$ a positive integer. The identity (1) will be used only in the two special cases when either
(A) $m=r-1, n=r$, or
(B) $m=r, n=r-1$.

Thus in either case $m+n=2 r-1$, and the functions $P(z), Q(z)$, and $R(z)$ take the following special forms. 
Case A:

$$
\begin{gathered}
P_{A}(z)=\sum_{k=r-1}^{2 r-1} k !(-1)^{k-1}\left(\begin{array}{c}
k \\
r-1
\end{array}\right)\left(\begin{array}{c}
2 r-1 \\
k
\end{array}\right) z^{2 r-k-1}, \\
Q_{A}(z)=\sum_{k=r}^{2 r-1} k !\left(\begin{array}{l}
k \\
r
\end{array}\right)\left(\begin{array}{c}
2 r-1 \\
k
\end{array}\right) z^{2 r-k-1}, \\
R_{A}(z)=\left(\begin{array}{c}
2 r-1 \\
r
\end{array}\right) z^{2 r} e^{z} \int_{0}^{1} w^{r-1}(w-1)^{r} e^{-z w} d \omega,
\end{gathered}
$$

and

Case B:

$$
\begin{gathered}
P_{B}(z)=\sum_{k=r}^{2 r-1} k !(-1)^{k-1}\left(\begin{array}{l}
k \\
r
\end{array}\right)\left(\begin{array}{c}
2 r-1 \\
k
\end{array}\right) z^{2 r-k-1}, \\
Q_{B}(z)=\sum_{k=r-1}^{2 r-1} k !\left(\begin{array}{c}
k \\
r-1
\end{array}\right)\left(\begin{array}{c}
2 r-1 \\
k
\end{array}\right) z^{2 r-k-1}, \\
R_{B}(z)=\left(\begin{array}{c}
2 r-1 \\
r
\end{array}\right) z^{2 r} e^{z} \int_{0}^{1} w^{r}(w-1)^{r-1} e^{-z w} d \omega .
\end{gathered}
$$

By these formulae, $P_{A}(z)$ and $Q_{B}(z)$ have the exact degree $r$, and $P_{B}(z)$ and $Q_{A}(z)$ have the exact degree $r-1$; further both $R_{A}(z)$ and $R_{B}(z)$ vanish at $z=0$ to the order $2 r$. Further, by (1),

$$
P_{A}(z) e^{z}-Q_{A}(z)=R_{A}(z) \text { and } P_{B}(z) e^{z}-Q_{B}(z)=R_{B}(z) \text {. }
$$

Therefore the determinant

$$
D(z)=\left|\begin{array}{ll}
P_{A}(z), Q_{A}(z) \\
P_{B}(z), Q_{B}(z)
\end{array}\right|=\left|\begin{array}{ll}
P_{A}(z), & -R_{A}(z) \\
P_{B}(z), & -R_{B}(z)
\end{array}\right|
$$

is a polynomial in $z$ of the exact degree $2 r$ which has at $z=0$ a zero of order $2 r$. This determinant can therefore be written as

$$
D(z)=d z^{2 r}
$$

where $d$ is a constant distinct from zero. Thus

$$
D(z) \neq 0 \text { if } z \neq 0 \text {. }
$$


All four polynomials $P_{A}(z), Q_{A}(z), P_{B}(z), Q_{B}(z)$ have integral coefficients divisible by $(r-1) !$, and they have the degrees $r$ or $r-1$ in $z$. Denote by $u$ and $v$ two positive integers and put in the preceding formulae

$$
z=u / v \text {. }
$$

Let further

$$
U_{A}=\frac{v^{r}}{(r-1) !} P_{A}(u / v), \quad V_{A}=\frac{v^{r}}{(r-1) !} Q_{A}(u / v), W_{A}=\frac{v^{r}}{(r-1) !} R_{A}(u / v)
$$

and

$$
U_{B}=\frac{v^{r}}{(r-1) !} P_{B}(u / v), \quad V_{B}=\frac{v^{r}}{(r-1) !} Q_{B}(u / v), \quad W_{B}=\frac{v^{r}}{(r-1) !} R_{B}(u / v)
$$

Then, by (2), $U_{A}, V_{A}, U_{B}, V_{B}$ are integers of determinant

$$
U_{A} V_{B}-U_{B} V_{A} \neq 0
$$

We require upper estimates for these six quantities and therefore introduce the two maxima

$$
X=\max \left(\left|U_{A}\right|,\left|V_{A}\right|,\left|U_{B}\right|,\left|V_{B}\right|\right) \text { and } Y=\max \left(\left|W_{A}\right|,\left|W_{B}\right|\right) .
$$

In the sum

$$
\sum_{k=0}^{2 r-1}\left(\begin{array}{c}
2 r-1 \\
k
\end{array}\right)=2^{2 r-1}
$$

the two terms with $k=r-1$ and $k=r$ are identical and hence satisfy the inequality

$$
\left(\begin{array}{c}
2 r-1 \\
r-1
\end{array}\right)=\left(\begin{array}{c}
2 r-1 \\
r
\end{array}\right) \leq 2^{2 r-2}
$$

It follows that also

$$
\frac{(2 r-1) !}{(r-1) !}=r !\left(\begin{array}{c}
2 r-1 \\
r-1
\end{array}\right) \leq 2^{2 r-2} r ! \text {. }
$$

Further, in the sums defining $U_{A}, V_{A}, U_{B}$, and $V_{B}$, the factors

$$
k !,\left(\begin{array}{l}
k \\
r
\end{array}\right) \text {, and }\left(\begin{array}{c}
k \\
r-1
\end{array}\right)
$$


assume their largest possible values when $k=2 r-1$. Hence trivially,

$$
X \leq \frac{(2 r-1) !}{(r-1) !}\left(\begin{array}{c}
2 r-1 \\
r-1
\end{array}\right)^{2}(u+v)^{r}
$$

and therefore

$$
X \leq 2^{6 r-6} r !(u+v)^{r} .
$$

Next, when $w$ lies in the interval $0 \leq w \leq 1$,

$$
\max \left(w^{r-1}(w-1)^{r}, w^{r}(w-1)^{r-1}\right) \leq(w(w-1))^{r-1} \leq 4^{-(r-1)} \text { and } e^{-z w} \leq 1 \text {. }
$$

The integrals for $W_{A}$ and $W_{B}$ imply therefore that

$$
Y \leq \frac{v^{r}}{(r-1) !} 2^{2 r-2}(u / v)^{2 r} e^{u / v_{4}-(r-1)}
$$

and hence that

$$
Y \leq \frac{e^{u / v}}{(r-1) !}\left(u^{2} / v\right)^{r}
$$

4.

These upper estimates will now be applied to the rational approximations of $e^{u / v}$. For this purpose, denote by $p$ and $q$ any two positive integers and put

$$
q e^{u / v}-p=d
$$

An explicit lower estimate for $|d|$ can be obtained by the following considerations.

Since the determinant (3) is distinct from zero, the same is true for at least one of the two determinants

$$
\left|\begin{array}{ll}
U_{A}, & V_{A} \\
q, & p
\end{array}\right| \text { and }\left|\begin{array}{cc}
U_{B}, & V_{B} \\
q, & p
\end{array}\right| \text {. }
$$

Denote then by $C$ the suffix $A$ or $B$ for which

$$
\left|\begin{array}{ll}
U_{C}, & V_{C} \\
q, & p
\end{array}\right| \neq 0 \text {. }
$$


The two equations

$$
U_{C} e^{u / v}-V_{C}=W_{C} \text { and } q e^{u / v}-p=d
$$

imply that

$$
p U_{C}-q V_{C}=d U_{C}-q W_{C}
$$

Here the left-hand side is an integer distinct from zero and is thus at least of absolute value 1 . Thus the following deduction can be made.

LEMMA. If the integer $r$ can be chosen such that

$$
\left|2 q W_{C}\right| \leq 1
$$

then also

$$
\left|2 d U_{C}\right| \geq 1
$$

5.

By the definition of $Y$ and by its upper estimate (5),

$$
\left|2 q W_{C}\right| \leq \frac{2 e^{u / v}}{(r-1) !}\left(u^{2} / v\right)^{r} \cdot q
$$

Assume now that $r$ satisfies the inequality

$$
(r-1) ! \geq 2 e^{u / v}\left(u^{2} / v\right)^{r} q,
$$

or, equivalently, the inequality

$$
e^{r} r ! \geq 2 r e^{u / v}\left(e u^{2} / v\right)^{r} q .
$$

Then the condition (6) holds, and it follows from (4) and (7) that

$$
|d| \geq\left(2^{6 r-5} r !(u+v)^{r \eta-1}\right. \text {. }
$$

To simplify these formulae, denote by $\varepsilon$ a constant in the interval

$$
0<\varepsilon \leq \frac{1}{2} \text {, }
$$

so that

$$
(1+2 \varepsilon)\left(1-\frac{\varepsilon}{2}\right)=1+\frac{3 \varepsilon}{2}-\varepsilon^{2}=1+\varepsilon+\varepsilon\left(\frac{1}{2}-\varepsilon\right) \geq 1-\varepsilon .
$$

Further assume from now on that both 
(10)

$$
q \geq\left(2 r e^{u / v}\left(e u^{2} / v\right)^{r}\right)^{1 / \varepsilon}
$$

and

$$
e^{r} r ! \geq q^{1+\varepsilon}
$$

Then the inequality ( 8 ) likewise is satisfied. Now almost trivially,

$$
r !>r^{r} e^{-r} \text {. }
$$

The hypothesis (11) may then be replaced by the following stronger one,

$$
r^{r} \geq q^{1+\varepsilon} \text {. }
$$

In order to satisy this condition, assume that $q$, in addition to the condition (10), also has the property that

$$
\log \log \log q \leq \frac{\varepsilon}{2} \log \log q,
$$

and then define $r$ as a function of $q$ by the equation

$$
r=\left[\frac{(1+2 \varepsilon) \log q}{\log \log q}\right]+1 \text {. }
$$

Then

$$
r>\frac{(1+2 \varepsilon) \log q}{\log \log q}
$$

and therefore

$$
\log r>\log \log q-\log \log \log q
$$

because $\log (1+2 \varepsilon)$ is positive. It follows that

$$
r \log r>\frac{(1+2 \varepsilon) \log q}{\log \log q}\left(1-\frac{\varepsilon}{2}\right) \log \log q \geq(1+\varepsilon) \log q .
$$

This shows that (12) is a consequence of the two formulae (13) and (14).

6.

Also the condition (13) may be replaced by a simpler one. If for the moment $c$ is any positive constant and $x$ a positive variable, the function

$$
x^{-c} \log x
$$


assumes its maximum at $x=e^{l / C}$ so that for all $x$,

$$
\log x \leq(c e)^{-1} x^{c} ;
$$

hence for $c=1 / 2$,

$$
\log x \leq(2 / e) x^{1 / 2}
$$

On putting $x=\log \log q$ in (13), this inequality takes the form

$$
\log x \leq \frac{\varepsilon}{2} x
$$

and is thus certainly satisfied if

$$
(2 / e) x^{1 / 2} \leq \frac{\varepsilon}{2} x \text {, that is, if } x \geq\left(\frac{4}{e \varepsilon}\right)^{2} \text {. }
$$

The condition (13) is thus a consequence of the simpler condition

$$
q \geq e^{e^{(4 / e \varepsilon)^{2}}}
$$

We have just replaced the condition (13) for $q$ by (16). As a next simplification, the other condition (10) for $q$ will now be replaced by two conditions in which the integer $r$ no longer occurs.

Evidently,

$$
2 r \leq e^{r}
$$

for all positive integers $r$. Hence the inequality (10) is certainly satisfied if

$$
q^{\varepsilon} \geq e^{u / v}\left(e^{2} u / v\right)^{r}
$$

Here, by (14),

$$
r \leq \frac{(1+2 \varepsilon) \log q}{\log \log q}+1
$$

Further

$$
0<\varepsilon \leq \frac{1}{2}, 1+4 \varepsilon \leq 3,\left(\frac{4}{e \varepsilon}\right)^{2}>8 .
$$

Since 


$$
\log \log q \geq\left(\frac{4}{e \varepsilon}\right)^{2},
$$

it follows therefore easily that

$$
r<\frac{3 \log q}{\log \log q}
$$

and so also

$$
\left(e^{2} u / v\right)^{r}<q^{\frac{3 \log \left(e^{2} u / v\right)}{\log \log q}}
$$

Hence the single inequality (17) for $q$ may be replaced by the pair of conditions

$$
q \geq e^{\left(e^{2} u / v\right)^{6 / \varepsilon}} \text { and } q \geq e^{2(u / v) / \varepsilon} \text {. }
$$

It depends on the size of $u / v$ which of these two conditions is the stronger one.

\section{7.}

By what so far has been proved, the three conditions (16) and (18) for $q$, together with the definition (14) of $r$ as a function of $q$, imply the inequalitiy (9) for $d$. This inequality still contains the parameter $r$, which will now be eliminated from it.

We found already that

$$
r \leq \frac{(1+2 \varepsilon) \log q}{\log \log q}+1
$$

Here $\log (1+x)<x$ for positive $x$, so that

$$
\begin{aligned}
\log x \leq \log \log q+\log (1+2 \varepsilon)- & \log \log \log q+\log \left(1+\frac{\log \log q}{(1+2 \varepsilon) \log q}\right) \leq \\
& \leq \log \log q-\log \log \log q+2 \varepsilon+\frac{\log \log q}{\log q} .
\end{aligned}
$$

Here, by (16),

$$
\log \log \log q \geq 2 \log \left(\frac{4}{e \varepsilon}\right) \geq 2 \log \frac{8}{e} \geq 2 \geq 4 \varepsilon
$$

and 


$$
\frac{\log \log q}{\log q} \leq\left(\frac{4}{e \varepsilon}\right)^{2} e^{-(4 / e \varepsilon)^{2}} \leq\left(\frac{4}{e \varepsilon}\right)^{2}\left(\frac{1}{6}\left(\frac{4}{e \varepsilon}\right)^{6}\right)^{-1} \leq 6\left(\frac{e \varepsilon}{4}\right)^{4}<\varepsilon .
$$

It follows that

$$
\varepsilon \frac{\log q}{\log \log q}>1
$$

whence

$$
r<\frac{(1+3 \varepsilon) \log q}{\log \log q}
$$

Further

$$
\log r \leq \log \log q-4 \varepsilon+2 \varepsilon-\varepsilon<\log \log q,
$$

so that

$$
r \log r<(1+3 \varepsilon) \log q
$$

and finally

$$
r ! \leq r^{r}<q^{1+3 \varepsilon} .
$$

On substituting this lower estimate for $r !$ in (9), it follows that

$$
|d|>32\left(\{64(u+v)\}^{r} q^{1+3 \varepsilon}\right)^{-1} \text {. }
$$

Here, by (19),

$$
\{64(u+v)\}^{r}<q^{\frac{(1+3 \varepsilon) \log \{64(u+v)\}}{\log \log q}} \leq q^{\frac{5 \log \{64(u+v)\}}{2 \log \log q}} .
$$

In order to simplify this estimate, add to the previous conditions (16) and (18) for $q$ the following new one,

$$
q \geq e^{\{64(u+v)\}^{5 /(2 \varepsilon)}} .
$$

The lower bound for $|d|$ takes then the simple form

$$
|d|>32 q^{-(1+4 \varepsilon)} \text {. }
$$

Here it is convenient to put

$$
4 \varepsilon=\delta
$$

Then the result just obtained may be formulated as follows.

THEOREM. Let $\delta$ be a constant in the interval $0<\delta \leq 2$, and let 
$p, q, u$, and $v$ be four positive integers where $q$ is restricted by the conditions

$q \geq e^{e^{(1 / e \delta)^{2}}}, q \geq e^{\left(e^{2} u / v\right)^{24 / \delta}}, q \geq e^{8(u / v) / \delta}, q \geq e^{\{64(u+v)\}^{10 / \delta}}$.

Then

$$
\left|e^{u / v}-\frac{p}{q}\right|>q^{-(2+\delta)}
$$

The conditions for $q$ in this theorem are stronger than necessary, and it would in particular be possible to replace the first condition by a weaker one. However, such a change would probably complicate the proof and the final result. My aim was to establish an effective lower bound for $\left|e^{u / v}-\frac{p}{q}\right|$ which does not contain any unknown constants.

\section{References}

[1] Kurt Mahler, "Zur Approximation der Exponentialfunktion und des Logarithmus, TeiI I", J. Reine angew. Math. 166 (1932), 118-136 (1931).

[2] Kurt Mahler, "Zur Approximation der Exponentialfunktion und des Logarithmus, Teil II", J. reine angew. Math. 166 (1932), 137-150.

[3] J. Popken, "Zur Transzendenz von e ", Math. 2. 29 (1929), 525-541.

Department of Mathematics, Institute of Advanced Studies, Australian National University, Canberra, ACT. 